\title{
Eingesandte Literatur
}

Die nachfolgenden Bücher sind zur Besprechung in LiLi eingeschickt worden. Die Herausgeber möchten darauf hinweisen, daß LiLi keinen regelmäßigen Rezensionsteil hat; jedoch ist vorgesehen, bei entsprechender Gelegenheit Sammelrezensionen, Forschungsberichte und Diskussionsrezensionen zu bringen.

Aguiar, Fernando: Minimal Poems. Visuelle Poesie 1991-1992. Universität-GH-Siegen, Siegen 1994 (experimentelle texte 38).

AUGEN-BLICK. Parallele Welten. Fallstudien zur deutschen Fernsehserie. Philipps-Universität Marburg, Marburg/Lahn 1994 (Marburger Hefte zur Medienwissenschaft 19).

Barck, Simone u.a. (Hrsg.): Lexikon sozialistischer Literatur. Ihre Geschichte in Deutschland bis 1945. Verlag J.B. Metzler, Stuttgart/ Weimar 1994.

Decher, Friedhelm: Die Ästhetik K. W. F. Solgers. Universitätsverlag C. Winter, Heidelberg 1994 (Reihe Siegen 125).

Diagonal. Zeitschrift der Universität-GH-Siegen. Zum Thema Fälschungen. Jg. 1994, Heft 2.

Eicher, Thomas; Ulf Bleckmann (Hg.): Intermedialität. Vom Bild zum Text. Aisthesis Verlag, Bielefeld 1994.

Erckenbrecht, Ulrich: Katzenköppe. Aphorismen/ Epigramme. Muri Verlag, Göttingen 1994.

Fachsprache. International Journal of LSP. 16. Jg., Heft 3-4/ 1994. Wilhelm Braumüller, Wien.

Faulstich, Werner; Helmut Korte (Hg.): Fischer Filmgeschichte, Bd. 5: Massenware und Kunst 1977-1995. Fischer Taschenbuch Verlag, Frankfurt/Main 1995.

Franzobel: Thesaurus. Ein Gleiches. 24 konzeptuelle Gedichte. Siegen 1995 (experimentelle texte 39).

Großmann, Rolf; Birgit Schlenther: Veröffentlichungen aus dem Sonderforschungsbereich >Bildschirmmedien< II. Universität-GH-Siegen, 2. aktualisierte Ausgabe, Siegen 1994 (Arbeitshefte Bildschirmmedien 51).

Hassauer, Friederike: Homo. Akademika. Geschlechterkontrakte, Institution und die Verteilung des Wissens. Passagen Verlag, Wien 1994 (Passagen Heft 10).

Hatvani, Paul: Die Ameisen. Mit einem Nachwort herausgegeben von Silke Hesse und Pavel Petr. Universität-GH-Siegen, Siegen 1994 (Vergessene Autoren der Moderne 62).

IASL. Internationales Archiv für Sozialgeschichte der deutschen Literatur. 19. Bd. 1994, Heft 2. Niemeyer Verlag, Tübingen 1994.

Jochmann, Carl Gustav: Briefe eines Homöopathischgeheilten an die zukünftigen Widersacher der Homöopathie. Nachdruck der Ausgabe Heidelberg 1829. Mit einem Nachwort herausgegeben von Uwe Pörksen. Universitätsverlag C. Winter, Heidelberg 1994.

Koch, Roland: Helle Nächte. Erzählungen. Verlag Kiepenheuer \& Witsch, Köln 1995.

Kreuzer, Helmut; Helmut Schanze (Hg.): »Bausteine III«. Beiträge zur Ästhetik, Pragmatik und Geschichte der Bildschirmmedien. Universität-GH-Siegen, Siegen 1994 (Arbeitshefte Bildschirmmedien 50).

Kübler, Hans-Dieter: Kommunikation und Massenkommunikation. Ein Studienbuch. LIT Verlag, Münster/Hamburg 1994 (Medien \& Kommunikation 21).

Kunkel, Roland: Schreibexperimente - Handreichung zum phantasiegeleiteten, literarischen Schreiben. Hessisches Institut für Bildungsplanung und Schulentwicklung, Wiesbaden 1994 (Kulturelle Praxis, Heft 2).

Langner, Margit: Sophie von La Roche. Die empfindsame Realistin. Universitätsverlag C.Winter, Heidelberg 1995 (Reihe Siegen. Beiträge zur Literatur-, Sprach- und Medienwissenschaft 126). 